\title{
Analyst
}

PAPER

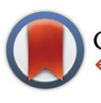

CrossMark

\&lick for updates

Cite this: Analyst, 2016, 141, 5374

\section{Fluorescence suppression using micro-scale spatially offset Raman spectroscopy}

\author{
Claudia Conti, ${ }^{\text {a }}$ Alessandra Botteon, ${ }^{a}$ Chiara Colombo, ${ }^{a}$ Marco Realini ${ }^{\mathrm{a}}$ and \\ Pavel Matousek*b
}

\begin{abstract}
We present a new concept of fluorescence suppression in Raman microscopy based on micro-spatially offset Raman spectroscopy which is applicable to thin stratified turbid (diffusely scattering) matrices permitting the retrieval of the Raman signals of sublayers below intensely fluorescing turbid over-layers. The method is demonstrated to yield good quality Raman spectra with dramatically suppressed fluorescence backgrounds enabling the retrieval of Raman sublayer signals even in situations where conventional Raman microscopy spectra are fully overwhelmed by intense fluorescence. The concept performance was studied theoretically using Monte Carlo simulations indicating the potential of up to an order or two of magnitude suppression of overlayer fluorescence backgrounds relative to the Raman sublayer signals. The technique applicability was conceptually demonstrated on layered samples involving paints, polymers and stones yielding fluorescence suppression factors between 12 to above 430 . The technique has potential applications in a number of analytical areas including cultural heritage, archaeology, polymers, food, pharmaceutical, biological, biomedical, forensics and catalytic sciences and quality control in manufacture.
\end{abstract}

\begin{abstract}
Received 12th April 2016, Accepted 13th June 2016 DOI: $10.1039 / c 6 a n 00852 f$ www.rsc.org/analyst
\end{abstract}

\section{Introduction}

Raman microscopy is a widely applicable technique used in numerous areas including art, archaeology, biomedicine and biology, polymer, food, forensics, semiconductors, pharmaceutics, geology and materials research and manufacture. In all these areas interfering fluorescence remains one of the key challenges posing major applicability limitations. Here we focus on addressing a common situation with turbid (diffusely scattering) stratified samples consisting of an intensely fluorescing, thin overlayer masking Raman signature of (not intensely fluorescing) sublayers, where the target of the analysis is the Raman signature of sublayers. This situation can often arise for example in art with paintings, frescoes or painted statues where the surface painted layer could be, due to its nature, environmental decay or ambient deposition processes, highly fluorescent precluding the analysis of the underlying layer(s) in a non-invasive way. Other examples from cultural heritage include the study of the substrate composition (stones, terracotta, stucco, plaster) hidden by an undesired

\footnotetext{
${ }^{a}$ Consiglio Nazionale delle Ricerche, Istituto per la Conservazione e la Valorizzazione dei Beni Culturali (ICVBC), Via Cozzi 53, 20125 Milano, Italy.

E-mail: conti@icvbc.cnr.it, pavel.matousek@stfc.ac.uk

${ }^{b}$ Central Laser Facility, Research Complex at Harwell, STFC Rutherford Appleton Laboratory, Harwell Oxford, OX11 OQX, UK
}

fluorescent over-layer of soot, atmospheric particulates or calcium oxalate films.

The masking of Raman spectra by fluorescence, in general, takes places via two processes which are of fundamental and instrumental origin: (i) the fluorescence imprints associated 'photon shot noise' on the overall Raman spectrum which can mask the present, weaker Raman signals. This noise is fundamental, inherently stemming from the dual nature of light (from the particle-like properties of photons) and follows Poisson statistics. ${ }^{1,2}$ Importantly, this noise is inseparable from the Raman signal by mathematical means once its source, fluorescence, is registered by the detection system. (ii) In addition, the detection of a high level of fluorescence also induces signal baseline distortions arising from the uneven sensitivity of the detection system to individual spectral components within the detected light. These distortions are instrumental in nature and increase linearly with the fluorescence background and at high levels can completely overwhelm Raman signals. The latter effect (but not the former) can be substantially reduced, or completely eliminated, by adopting. advanced detection approaches such as Shifted-Excitation Raman Difference Spectroscopy (SERDS) and its derivatives or by mathematical subtraction with the spectral distortions accurately measured $a$ priori. ${ }^{3}$ Such methods however are of limited effectiveness and, in addition, they cannot deal with the primary noise mechanism, the photon shot noise, which is of fundamental nature. 
In general, the most effective suppression methods are those where the background distortions and photon shot noise are both avoided or minimised by reducing the level of fluorescence photons registered by the detector. The most common class of methods belonging to this category is temporal rejection of fluorescence. This relies on impulsive excitation of the Raman signal and gated detection exploiting differences between the fluorescence lifetime and Raman emission timescales, the latter being, for most practical purposes, instantaneous (i.e. within the time scale of the interaction of the laser pulse with the sample). ${ }^{1,3,4}$ Another example is fluorescence bleaching ${ }^{5}$ which is commonly practiced with Raman microscopy although this approach can have a varying degree of effectiveness and involves chemical alteration of the sample, by its nature, a potentially undesirable feature in some situations. Another method is spatially gated suppression of fluorescence in stratified turbid matrices reported in several publications ${ }^{6-11}$ with conventional macroscale spatially offset Raman spectroscopy (SORS). ${ }^{12}$ In these situations only basic observations of suppression were reported with no systematic studies carried out. For example, Matousek ${ }^{6}$ reported the observation of the suppression of fluorescence with envelopes containing sugar powder. Olds et al. ${ }^{7}$ reported the use of SORS in the analysis and detection of packaged pharmaceuticals and concealed drugs where fluorescence suppression was observed with padded envelopes containing powder (barium sulfate) and pharmaceutical capsules in blister packs. Eliasson and Matousek $^{9}$ also reported fluorescence suppression with pharmaceutical capsules. Fluorescence suppression was also noted in the work of Matousek et $a l .{ }^{8}$ on non-invasive Raman spectroscopy of human bone in vivo where suppression of fluorescence from the skin layer, predominantly due to melanin, was also observed with SORS.

Here we demonstrate that the micro-Spatially Offset Raman Spectroscopy (micro-SORS) method ${ }^{13-15}$ is also a viable technique for fluorescence rejection. The technique combines Raman microscopy with Spatially Offset Raman Spectroscopy (SORS) and addresses the measurement of thin turbid layers. As this concept reduces the level of fluorescence relative to the Raman signal registered by the detection system the method belongs to the category of the abovementioned more effective methods that suppress both the photon shot noise and fluorescence background distortions. This is due to the fact that non-removable 'photon shot' noise associated with fluorescence backgrounds is not acquired by the detection system in the first place as the fluorescence background itself is rejected before entering the detection system. As a SORS method, in general, the concept relies on the diffuse component of light ${ }^{16}$ in analogy with techniques used in NIR absorption tomography ${ }^{16-18}$ and fluorescence spectroscopy. ${ }^{19-22}$

It should also be noted that previous work on fluorescence suppression using SORS concerned only macro-scale SORS and the applicability of this concept to micro-scale SORS, i.e. up to three orders of magnitude shorter space scales, does not involve necessarily straightforward transformation of technology. This is due to the fact that the process is complex and depends on several parameters including the dimensions of layers, laser illumination area, Raman collection area, the magnitude of spatial offset, mean free scattering path lengths of photons in the layers and the absorption coefficients of layers. Although the underlying physical processes are scalable spatially, in practice not all the parameters necessarily change by the same magnitude on the transformation from the macro-regime to the micro-regime and as such an entirely different regime can be present on the micro-scale. This could lead to different efficacy of the fluorescence suppression or even to its lack on the micro-scale. As such a separate investigation of the process on the micro-scale in practically relevant situations is required.

The fluorescence suppression using micro-SORS is demonstrated and characterised here for two principal variants of micro-SORS: (i) defocusing micro-SORS and (ii) full microSORS $^{23}$ (see Fig. 1). The defocusing variant involves measurements performed at several defocusing distances from the 'imaged' position (where the sample surface is sharply imaged). The defocusing leads to the enlargement of both the collection and illumination points and the invoking of the SORS effect. ${ }^{15}$ In contrast, full micro-SORS mimics the classical macro-scale SORS effect on the micro-scale using fully sep-

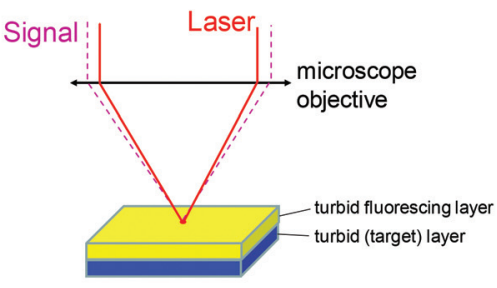

a) Imaged position (conventional Raman)

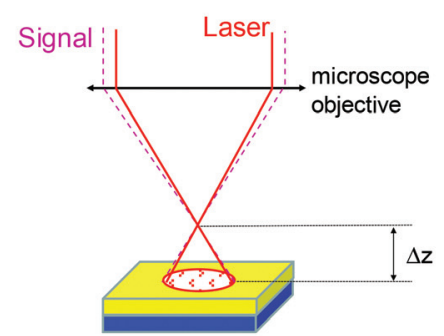

b) Defocusing micro-SORS

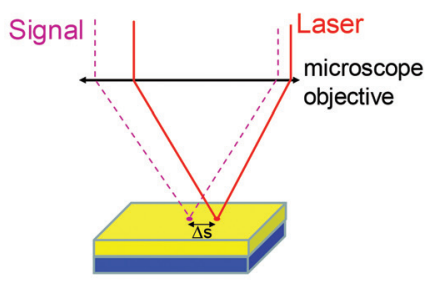

c) Full micro-SORS

Fig. 1 Schematic diagram of (a) conventional Raman microscopy of the turbid stratified sample ('imaged' position), (b) defocusing micro-SORS and (c) full micro-SORS in a situation with a highly fluorescing overlayer followed by a target layer from which the Raman signal is desired (Raman signal - dashed line, laser beam - solid line). 
arated Raman collection and laser illumination zones. Although the defocusing micro-SORS is a less effective variant compared with the fully separated micro-SORS it is exceptionally simple and can be practised on existing Raman microscopes without any modifications, unlike the full micro-SORS, which requires adaptations in order to be practised in its unrestricted form. ${ }^{15,24}$ The instrumental modification penalty required for the full micro-SORS is offset by its much higher degree of surface fluorescence suppression capability. For this reason both the methods are included in this study and compared both theoretically and experimentally.

In general, with both the methods the introduction of defocusing $\Delta z$ or spatial offset $\Delta s$ (see Fig. 1) leads to the suppression of the fluorescence signal from surface layers and the enhancement of the contrast of sublayer Raman signals. A sufficiently clear signature of the sublayer Raman signal could either be directly obtained at a certain magnitude of defocusing spatial offset or alternatively the fluorescence background obtained at the imaged zero-offset position can be used and the spectrum scaled was subtracted away from the spectrum obtained at a defocused spatially offset position to reveal the clear Raman signature of the sublayer removing any residual fluorescence and reducing the associated background distortions. It should be recognised that the degree of fluorescence suppression is limited and is expected to be also different with the two approaches. As such, in some situations, where extreme fluorescence is present, one or both of these concepts may not be potent enough to reveal sublayer Raman signals.

\section{Monte Carlo simulations}

Monte Carlo simulations were used to characterise the fluorescence rejection properties of both the defocusing and fully separated micro-SORS variants. The sample was assumed to consist of two thin turbid layers located on top of an extended turbid substrate. For simplicity the transport length $t$ was assumed to be the same for all the three layers and no excessive absorption of laser photons was present apart from that leading to the weakly generated Raman and fluorescence signals. The top layer was assumed to yield a fluorescence signal upon incidence by laser photons whereas the second and third layers were purely Raman emissive. No significant absorption was assumed to be present in any of the layers for all the laser, fluorescence and Raman emission wavelengths. The simulations were carried out using a Raman propagation code described earlier, as both fluorescence and Raman processes manifest themselves in analogous ways in (micro-)SORS measurements. $^{23,25}$ The laser photons, fluorescence and Raman photons were individually tracked through a turbid medium in three dimensions with each calculation propagation step length equated to the photon transport length $t$, i.e. the distance at which the photon direction of travel is just fully randomised. ${ }^{16}$ This model was shown to predict well photon behaviour on propagation distances much larger than the photon transport length such as those encountered in common SORS situations. ${ }^{25}$
In the defocusing method the beam diameter of the incident laser beam and the collection Raman areas at the sample surface, defined typically by the numerical aperture of the microscope objective and the degree of defocusing, were of an identical diameter $10 \mu \mathrm{m}$ and exactly overlapped at the 'imaged' position.

For practical purposes, with the full micro-SORS method the Raman photon collection was assumed to be facilitated through a ring collection zone area of a constant thickness of $10 \mu \mathrm{m}$ drawn around the illumination zone and being concentric with it. The laser illumination zone diameter was also kept constant $(10 \mu \mathrm{m})$. The spatial offset was defined as the radius of the Raman collection ring taken from the ring centre and its midpoint. The results with this concept also replicate the performance of a point-like collection geometry used in the subsequent experimental demonstration for spatial offsets much larger than the laser beam and Raman collection zone radii. (The use of the ring illumination zone was necessitated by the nature of Monte Carlo simulations performed here to facilitate manageably short simulation times by yielding much higher numbers of 'detected' Raman and fluorescence photons than the point collection geometry would otherwise do.)

For each value of the defocusing diameter or spatial offset the number of detected Raman photons originating from each layer was evaluated. The laser beam was assumed to be of uniform intensity across its spatial profile at the point of incidence. 2000000 photons were propagated simultaneously through 5000 steps, each step was equated to the photon transport length $t$, which was set to $5 \mu \mathrm{m}$ in all calculations and was assumed to be the same for all the three layers (corresponds to the reduced scattering coefficient $\mu_{\mathrm{s}}=0.2 \mu \mathrm{m}^{-1}$ ). The photon transport length chosen crudely represents a value one could encounter with art pigments. ${ }^{26,27}$

Fig. 2 illustrates achievable theoretical fluorescence suppression factors (the reduction of the ratio between the Raman band and fluorescence background intensities) for both thin and thick sublayers below the intensely fluorescing overlayer for the full micro-SORS and defocusing micro-SORS. Both the methods provide significant suppression factors although the full micro-SORS concept is far superior in terms of performance. For the thin sublayer with a $300 \mu \mathrm{m}$ spatial offset defocusing diameter the full micro-SORS yields fluorescence suppression of $\times 58$ as opposed to $\times 7.9$ achievable with the defocusing method. That is full micro-SORS outperforms the defocusing concept by a factor of $\times 7.3$. For the thick sublayer with a $300 \mu \mathrm{m}$ spatial offset defocusing diameter the full micro-SORS yields fluorescence suppression of $\times 190$ as opposed to $\times 11$ achieved by the defocusing method. That is full micro-SORS outperforms the defocusing concept by a factor of $\times 17$. These are highly effective fluorescence suppression factors predicted by theory making potentially a difference, for example, between detecting the Raman signal and not sensing it at all. It should be noted that the performance will vary depending on the actual optical properties of the specific sample and its layer thicknesses. These general findings 


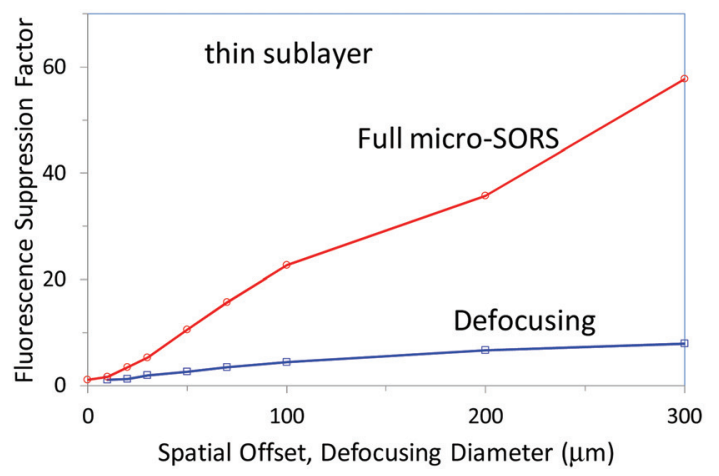

b

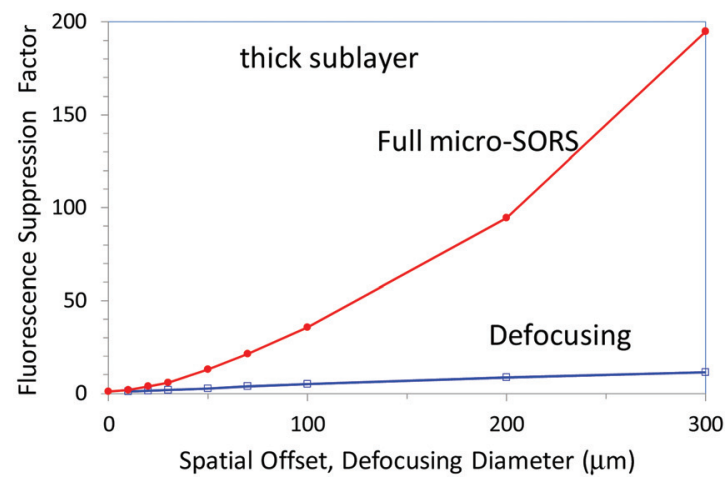

Fig. 2 The fluorescence suppression factors for the Raman sublayer signal derived using Monte Carlo simulations (a) from a thin (second) sublayer ( $40 \mu \mathrm{m}$ thick) below a $20 \mu \mathrm{m}$ thick fluorescent overlayer with both layers being deposited on top of an extended diffusely scattering substrate and (b) from an extended turbid sublayer below a $20 \mu \mathrm{m}$ thick fluorescent over-layer achieved using full and defocusing micro-SORS methods.

are also consistent with our experimental findings presented below.

\section{Experimental}

\section{Defocusing micro-SORS}

The measurements were carried out using a Senterra dispersive micro-Raman spectrometer (Bruker Optik $\mathrm{GmbH}$ ) that includes a standard confocal optical microscope (Olympus BX51). The Raman spectra were acquired using a $20 \times$ objective (WD $1.3 \mathrm{~mm}$, NA 0.4) and a laser $785 \mathrm{~nm}$ excitation wavelength with a nominal power of $100 \mathrm{~mW}$. The detection system consisted of a Peltier cooled CCD detector $(1024 \times 256$ pixels $)$, and 1200 grooves $\mathrm{mm}$ grating and the largest confocal slit $(50 \mu \mathrm{m} \times$ $1000 \mu \mathrm{m})$ was used. The spectra were acquired with an acquisition time of $10 \mathrm{~s}$ and 20 accumulations (i.e. the total acquisition time per sample position was $200 \mathrm{~s}$ ). The combined sample illumination and collection area at the 'imaged' position with the $20 \times$ objective was estimated experimentally to be approximately $4 \mu \mathrm{m}$ in diameter.

Measurements were acquired in different sample positions, (i) first, with the sample in the 'image' plane (the 'zero' position) and then (ii) with the sample displaced away the microscope objective along the microscope objective axis by 'defocusing distances $\Delta z$ ' to induce enlargement of laser illumination and Raman collection zones on the sample surface.

\section{Full micro-SORS}

The measurements were carried out using the same instrumentation with a small modification. An external standard Raman probe (UniLabII, Bruker Optik $\mathrm{GmbH}$ ), equipped with a $4 \times$ lens (working distance about $15 \mathrm{~mm}$ ), was used to deliver the laser beam to the sample (by passing the microscope optics) and directed at about $50^{\circ}$ with respect to the incidence plane to the sample. This resulted in a somewhat elongated illumination spot on the sample surface. The elongation direction was set perpendicular to the direction of the spatial offset direction to minimise its influence on the SORS measurements. In this configuration its effect would only be noteworthy at the zero ('imaged') position or at very small spatial offsets. The laser beam spot size on the sample surface was determined by performing Raman mapping across a sharp edge of a silicon reference sample, moving the reference sample by the automated sample stage with the probe fixed in its position on an external mount. It was measured to be approximately $30 \mu \mathrm{m}$ along the $x$ axis and $40-50 \mu \mathrm{m}$ along the $y$ (elongated) axis (calculated between $90 \%$ and $10 \%$ of the maximum signal). The Raman signal was collected using the standard microscope optics and a $4 \times$ objective (WD $18.5 \mathrm{~mm}$, NA 0.1). The external probe was mounted on the sample micro-positioning stage (OPUS-IR ${ }^{(\mathrm{TM})}$ software controlled) enabling controlled sideways movement to permit the setting of spatial offsets with respect to the Raman collection zone with a high accuracy and reproducibility in the positioning (i.e. mapping measurements were carried out by moving the laser leaving the sample at the same position). To keep the sample immobile with respect to the objective during the measurements it was positioned on top of the condenser, used as a fixed sample stage. Raman spectra were acquired at different spatially offset distances (in the range from $100 \mu \mathrm{m}$ to $1 \mathrm{~mm}$ ) with an acquisition time of $10 \mathrm{~s}$ and 20 accumulations each (i.e. the total acquisition time per offset position was $200 \mathrm{~s}$ ).

\section{Specimens}

The first specimen (S1) comprised an artificially assembled two-layer system: the top layer was a fluorescent brown paper sheet $(80 \mu \mathrm{m}$ thick) placed over a $1 \mathrm{~mm}$ thick sublayer of a plastic made of polystyrene. Both layers were highly turbid and opaque in appearance. The second artificially prepared specimen (S2) consisted of a fluorescent yellow paper sheet $(120 \mu \mathrm{m}$ thick) followed by a painted layer made of phthalocyanine blue $\left(\mathrm{C}_{32} \mathrm{H}_{16} \mathrm{~N}_{8} \mathrm{Cu}-80 \mu \mathrm{m}\right.$ thick $)$ in acrylic media deposited on a sheet of paper. The third example (S3) simulates a real artistic stratigraphy: cadmium red PR108 pigment (cadmium selenosulphite) was spread with a thickness of $\sim 40 \mu \mathrm{m}$ on a painted 
Table 1 Investigated samples

\begin{tabular}{lll}
\hline Sample & Top layer & Bottom layer \\
\hline $\begin{array}{ll}\text { Paper on } \\
\text { plastic (S1) }\end{array}$ & Brown paper & $\begin{array}{l}\text { Polystyrene } \\
(1000 \mu \mathrm{m})\end{array}$ \\
Paper on & Yellow paper & $\begin{array}{l}\text { Phthalocyanine } \\
\text { blue }(80 \mu \mathrm{m})\end{array}$ \\
pigment $(\mathrm{S} 2)$ & $(120 \mu \mathrm{m})$ & Phthalocyanine \\
Painted & Cadmium red & blue $(80 \mu \mathrm{m})$ \\
stratigraphy (S3) & PR108 $(40 \mu \mathrm{m})$ & Marble - calcite \\
Pigment on & Cadmium red & $(20000 \mu \mathrm{m})$ \\
marble (S4) & PR108 $(30 \mu \mathrm{m})$ &
\end{tabular}

layer made of phthalocyanine blue $\left(\mathrm{C}_{32} \mathrm{H}_{16} \mathrm{~N}_{8} \mathrm{Cu}-80 \mu \mathrm{m}\right.$ thick) deposited on a sheet of paper. Both pigments are mixed in acrylic media. As the fourth example (S4), the same cadmium red PR108 pigment was spread with $\sim 30 \mu \mathrm{m}$ on a marble stone ( $2 \mathrm{~cm}$ thick), mainly consisting of calcite $\left(\mathrm{CaCO}_{3}\right)$ (Table 1$)$.

\section{Results and discussion}

The first demonstration was carried out on a stratified system (S1) composed of polystyrene plastic covered with an 80 micrometre thick fluorescent brown paper. Fig. 3 shows the results of these measurements. The full sequence of spectra for different spatial offsets for full micro-SORS is shown on a logarithmic scale to convey the large change in the fluorescence background induced. The full micro-SORS spectrum at "0" offset ('imaged') yields an intense fluorescence background with no discernible Raman bands assignable to the sublayer. Only background distortion and spurious bands or bands belonging to the surface layer can be seen upon a visual inspection in the high intensity fluorescence background. As the laser beam is offset from the collection point, the fluorescence of the top layer evidences a dramatic decrease in intensity and the Raman signal from the sublayer starts to become clearly identifiable through its characteristic band at $\sim 1000 \mathrm{~cm}^{-1}$ (see Fig. 3a). The fluorescence is most suppressed with 800 and $1000 \mu \mathrm{m}$ spatial offsets. The contrast improvement of the Raman intensity to the fluorescence background intensity (fluorescence suppression factor) was estimated to be in excess of $\times 110$ for $800 \mu \mathrm{m}$ spatial offset. The actual contrast improvement factor could not be determined in this instance as no Raman bands from the sublayer were detected at the imaged position. As such this value is based only on the undetected Raman signal at the imaged position that must have been smaller than the neighbouring background (fluorescence) noise level. At larger offsets the entire Raman spectrum of the sublayer can clearly be seen. Defocusing microSORS results are not shown as these permitted much lower suppression of the top-layer fluorescence relative to the sublayer insufficient to permit the detection of the sublayer in the studied range of 50 to $1000 \mu \mathrm{m}$ of defocusing distances $\Delta z$.

To recover the pure Raman spectrum of the sub-layer, the micro-SORS "0" offset spectrum was subtracted from the offset one (see Fig. 3b). This was performed for both full and defocusing micro-SORS measurements. For the full micro-SORS measurements, the resulting pure Raman component of the bottom layer compares well with the reference spectrum of this layer. However, no traces of the sublayer Raman signal were observed neither in the subtracted defocusing microSORS spectra nor in the purely baseline subtracted 'image' spectrum acquired in conventional Raman geometry. This is in line with our theoretical modelling predicting particularly large fluorescence suppression factors for the full micro-SORS concept and much smaller for the defocusing concept, which in this particular case did not yield sufficient contrast enhancement to reveal any sublayer Raman bands in the pertinent intense fluorescence scenario. In considerably less severe situations defocusing micro-SORS would be expected to render a sublayer spectrum too.
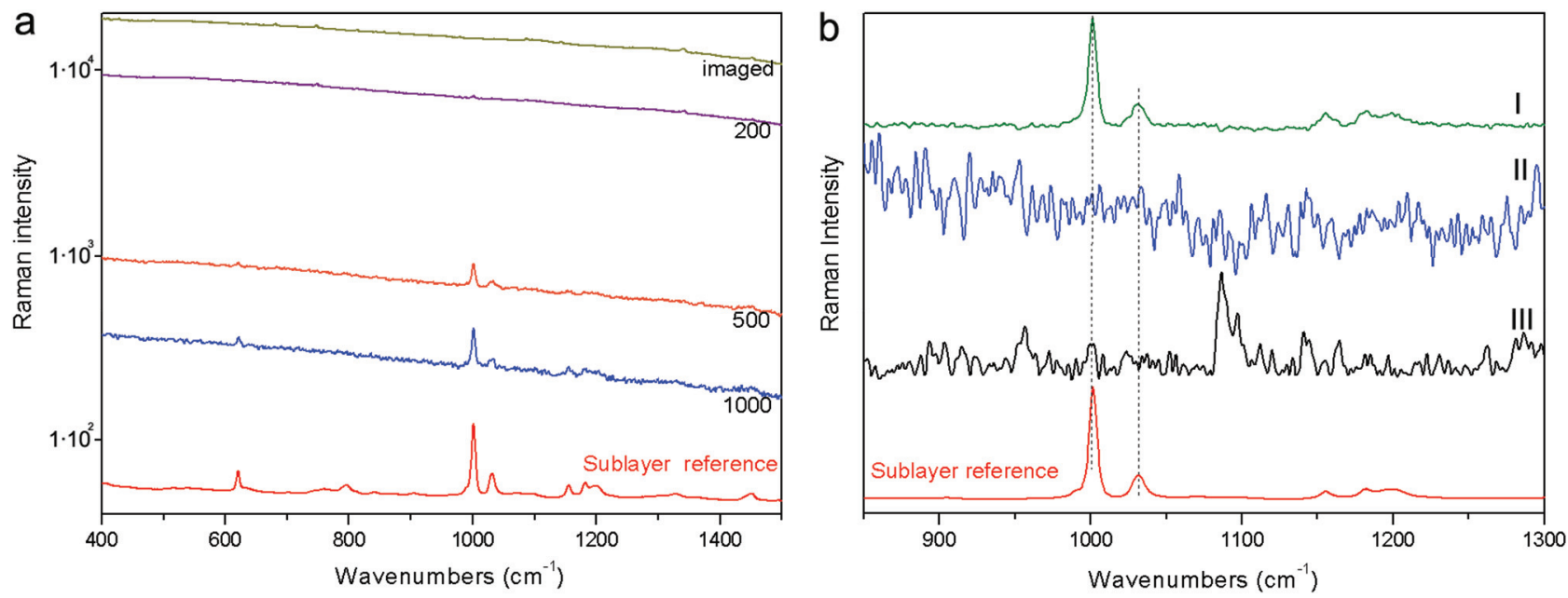

Fig. 3 S1: full micro-SORS (a) sequences. Spatial offsets in $\mu \mathrm{m}$ are indicated next to the spectra. The spectra are plotted on a logarithmic scale. Subtraction results (b); full micro-SORS subtraction between 'imaged' and $1000 \mu \mathrm{m}$ offset (I), defocusing subtraction between imaged and $1000 \mu \mathrm{m}$ defocusing distance (II) and conventional Raman imaged baseline subtracted (III). Reference spectra are shown in red. 

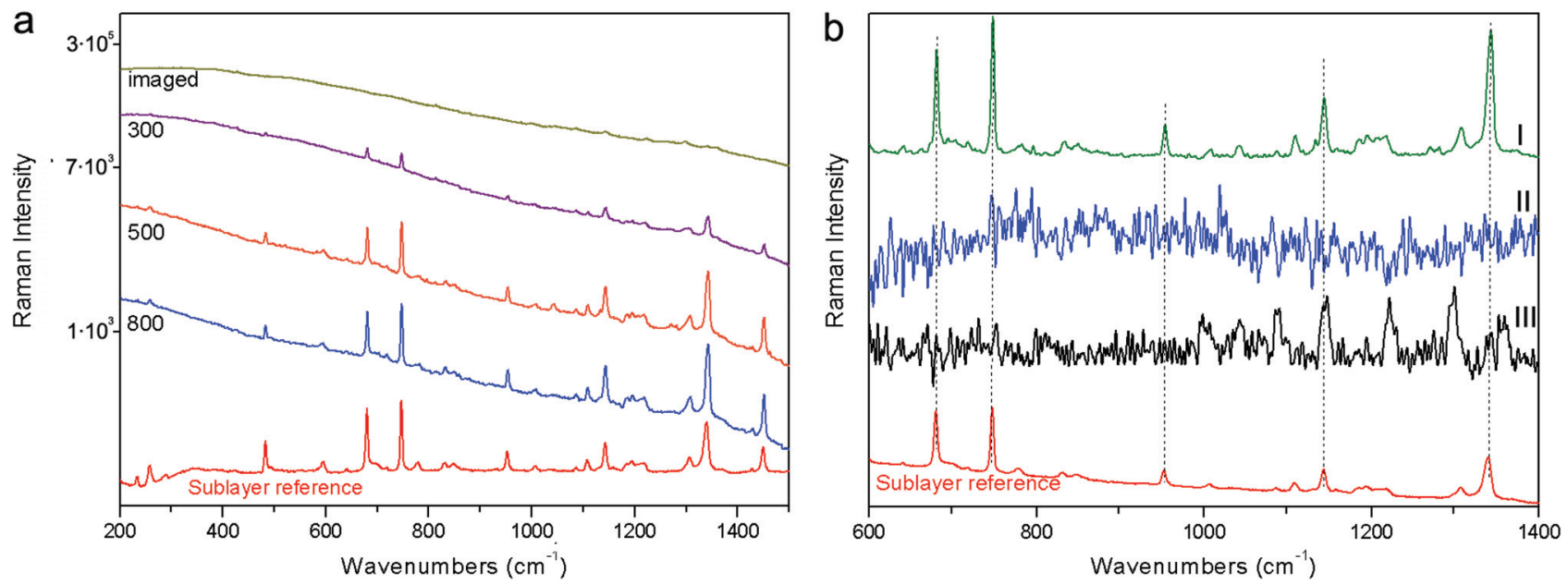

Fig. 4 S2: full micro-SORS (a) sequences. Spatial offsets in $\mu \mathrm{m}$ are indicated next to the spectra. The spectra are plotted on a logarithmic scale. Subtraction results (b); full micro-SORS subtraction between 'imaged' and $800 \mu \mathrm{m}$ offset (I), defocusing subtraction between imaged and $1000 \mu \mathrm{m}$ defocusing distance (II) and conventional Raman imaged baseline subtracted (III). Reference spectra are shown in red.

Another example of fluorescence suppression is given on a two layer system, composed of a sub-layer of the phthalocyanine blue pigment covered by a thin film of highly fluorescent yellow paper (S2). Selected full micro-SORS spectra from the zero to $800 \mu \mathrm{m}$ positions are shown on a logarithmic scale in Fig. 4. Here a dramatic suppression of the top layer fluorescence and the relative increase of the sublayer signal with full micro-SORS are again clearly evidenced. The contrast improvement of the Raman intensity to the fluorescence background level was at least $\times 160$ (again no Raman band was visible at the imaged position and therefore this value is estimated from the noise level at the imaged position). The characteristic bands of the phthalocyanine blue pigment arise at $300 \mu \mathrm{m}$ offset and their intensity increases up to $800 \mu \mathrm{m}$ offset. By applying the same scaling method to the two raw spectra one is able to recover the estimates of the pure Raman sublayer component (see Fig. 4a); the spectrum of the sublayer recovered from full micro-SORS compares well with the reference spectrum. The defocusing measurement again did not permit the recovery of the sublayer signal. Also the pure subtraction of the baseline from the conventional, ' 0 ' spatial offset spectrum ('imaged') did not reveal any underlying Raman bands either (see Fig. 4b). Only baseline distortion and Raman bands belonging to the surface layer can be seen in this case.

The third specimen (S3) consisted of a red pigment layer deposited on a blue paint layer. The red pigment exhibits a strong fluorescence that effectively masks the Raman signal of the sublayer. As seen in Fig. 5a the full micro-SORS spectra at the "imaged" position are dominated by the fluorescence signal from the top layer, while phthalocyanine blue, the main
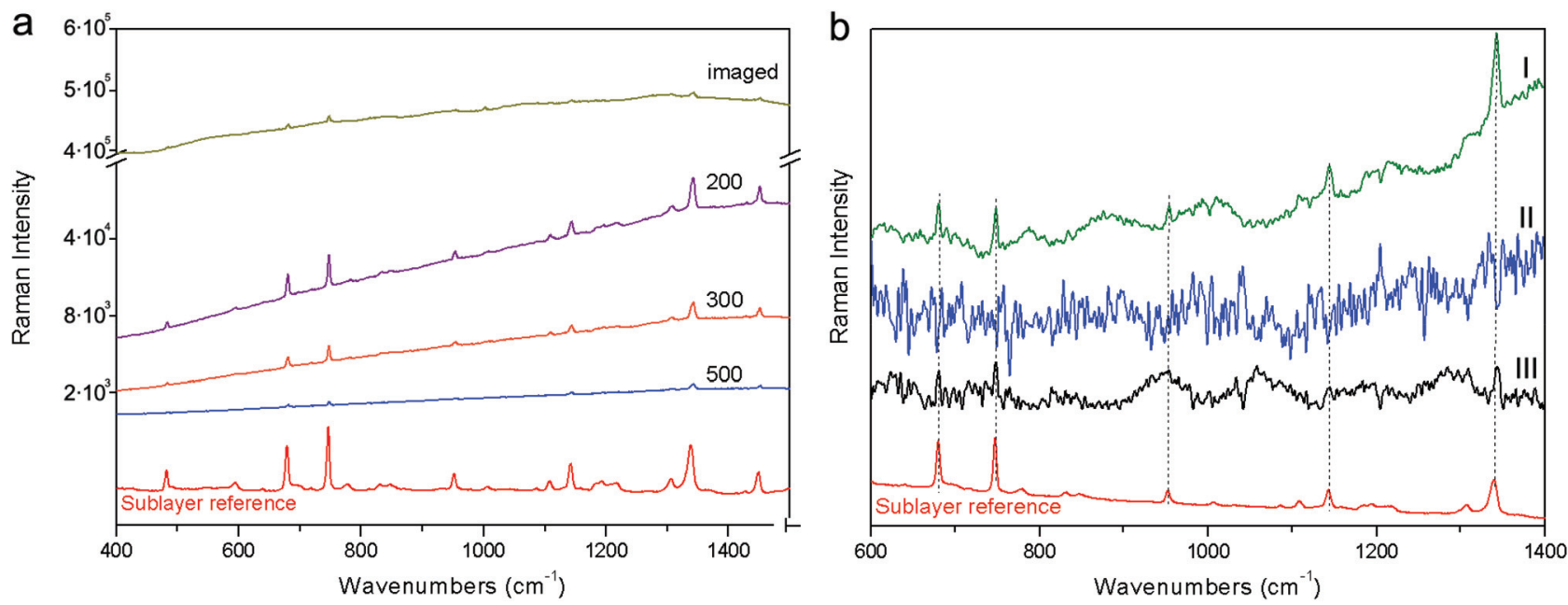

Fig. 5 S3: full micro-SORS (a) sequences. Spatial offsets in $\mu \mathrm{m}$ are indicated next to the spectra. Subtraction results (b); full micro-SORS subtraction between 'imaged' and $200 \mu \mathrm{m}$ offset (I), defocusing subtraction between imaged and $1000 \mu \mathrm{m}$ defocusing distance (II) and conventional Raman imaged baseline subtracted (III). Reference spectra are shown in red. 
component of the bottom layer, is just about detectable through very small peaks arising from the background. Moving the laser beam sideways in the full micro-SORS geometry improves the contrast of the sublayer very effectively: the increase of phthalocyanine blue is unequivocally observed from the zero to $200 \mu \mathrm{m}$ spatial offsets. The contrast improvement of the Raman intensity to the fluorescence background intensity was estimated to be $\times 12$. A further increase of spatial offset leads to an absolute decrease of the sublayer Raman signal intensity. In general, the Raman signal of the sublayer in absolute terms decreases with the increasing spatial offset for all layers present in full micro-SORS, but with a different rate for each of them with the fastest decay rate exhibited by the top layer. As such the contrast improves for sublayers but the absolute signal still decreases monotonically with the increasing spatial offset. This leads to the existence of an optimum offset rendering the recovered pure sublayer signal with the largest signal-to-noise ratio. This can be determined experimentally or theoretically, the latter in situations where optical properties of the layers are known. ${ }^{28}$

In this case the Raman bands of the sublayer were visible in the imaged spectrum permitting the determination of the actual contrast enhancement factor in this situation. On the contrary, the increase of defocusing distances did not provide an adequate contrast change between the fluorescence and sublayer signal to enable the recovery of the sublayer signal by this approach. The Raman spectra of the sublayer recovered from the full micro-SORS measurements by scaled subtraction are shown in Fig. 5b. Again, the result is in good agreement with the reference spectrum; due to the presence of small peaks of phthalocyanine blue at the "imaged" positions, even the baseline subtracted image spectrum acquired in conventional geometry yielded the Raman band of the sublayer in reasonable agreement with the reference spectrum, while no sublayer signals were observed in the subtracted defocusing spectra due to the lack of the adequate degree of contrast change using defocusing with this sample configuration.

The final example (S4) consisted of the same red pigment 30 micrometre layer as above spread on top of a marble substrate. The evolution of the signals for full micro-SORS with spatial offset is shown on a logarithmic scale in Fig. 6. In this case the conventional Raman spectrum ('imaged') is completely overwhelmed by the fluorescence signal with no visible Raman signal due to the sublayer being seen. The increase of the spatial offset with full micro-SORS again led to the dramatic contrast enhancement of the Raman signal from the sublayer permitting the clear observation of the sublayer Raman spectrum. The contrast improvement was estimated to be at least $\times 430$ (no Raman band was visible at the imaged position and therefore this value is estimated from the noise level at the imaged position). The subtraction of full SORS spectra yielded the pure Raman spectrum of the sublayer with excellent quality. Whilst no Raman signal could be recovered using defocusing SORS nor from a simple subtraction of the polynomial baseline from the conventional (imaged) spectrum.

The above examples illustrate the superiority and effectiveness of the full SORS method in the non-invasive recovery of the Raman sublayer signal in the presence of intensely fluorescing overlayers, even in situations where the conventional Raman spectrum is completely overwhelmed by the fluorescence background and traditional polynomial baseline subtraction does not yield any Raman signature from the sublayer. The achieved fluorescence suppression factors ranging from one to two orders of magnitude are in broad agreement with theoretical predictions. The method has naturally its limitations which include inapplicability to highly absorbing layers or situations involving highly fluorescing sublayers.
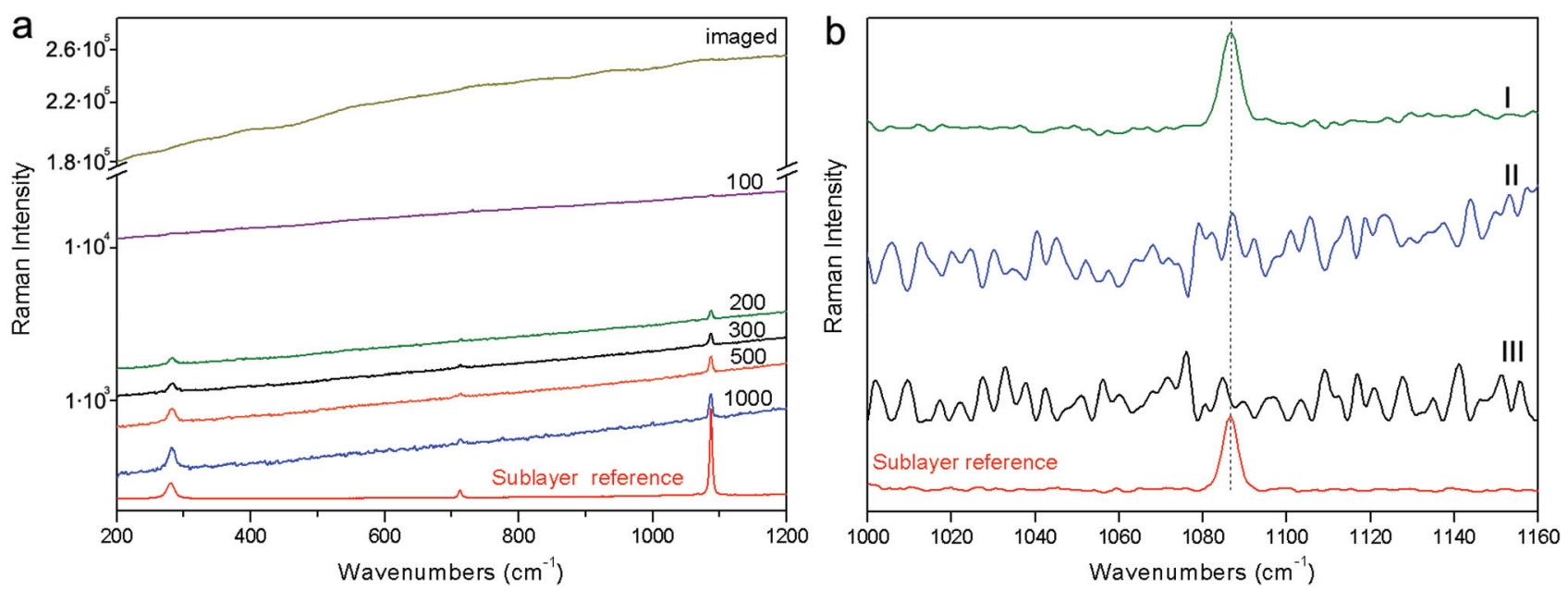

Fig. 6 S4: full micro-SORS (a) sequences. Spatial offsets in $\mu \mathrm{m}$ are indicated next to the spectra. The spectra are plotted on a logarithmic scale. Subtraction results (b); full micro-SORS subtraction between 'imaged' and $1000 \mu \mathrm{m}$ offset (I), defocusing subtraction between imaged and $1000 \mu \mathrm{m}$ defocusing distance (II) and conventional Raman imaged baseline subtracted (III). Reference spectra are shown in red. 


\section{Conclusions}

We have presented and demonstrated a new concept of fluorescence suppression applicable to Raman microscopy with thin turbid stratified samples where intense fluorescence from an overlayer masks the Raman signals of sublayers. Both theoretical and experimental validation of the concept is presented here. The results of Monte Carlo simulations indicate that fluorescence suppression factors up to an order or two of magnitude are achievable with this method. This has been validated by a proof-of-concept study in which Raman signals were recovered from intense fluorescence backgrounds even in situations where these were completely masked by fluorescence in conventional Raman microscopy measurement. Both the theoretical and experimental results evidence that full micro-SORS is superior in its performance to defocusing micro-SORS. The concept has a wide range of potential applications including cultural heritage, archaeology, polymers, food, pharmaceutical, forensics and catalytic sciences and quality control in manufacture.

\section{References}

1 P. Matousek, M. Towrie and A. W. Parker, J. Raman Spectrosc., 2002, 33, 238-242.

2 P. Matousek, Chem. Soc. Rev., 2007, 36, 1292-1304.

3 D. Wei, S. Chen and Q. Liu, Appl. Spectrosc. Rev., 2015, 50, 387-406.

4 P. Matousek, M. Towrie, A. Stanley and A. W. Parker, Appl. Spectrosc., 1999, 53, 1485-1489.

5 Emerging Raman Applications and Techniques in Biomedical and Pharmaceutical Fields, ed. P. Matousek and M. D. Morris, Springer, Heidelberg, 2010.

6 P. Matousek, Appl. Spectrosc., 2006, 60, 1341-1347.

7 W. J. Olds, E. Jaatinen, P. Fredericks, B. Cletus, H. Panayiotou and E. L. Izake, Forensic Sci. Int., 2011, 212, 69-77.

8 P. Matousek, E. R. C. Draper, A. E. Goodship, I. P. Clark, K. L. Ronayne and A. W. Parker, Appl. Spectrosc., 2006, 60, 758-763.

9 C. Eliasson and P. Matousek, Anal. Chem., 2007, 79, 16961701.
10 N. Stone, R. Baker, K. Rogers, A. W. Parker and P. Matousek, Analyst, 2007, 132, 899-905.

11 N. A. Macleod and P. Matousek, Appl. Spectrosc., 2008, 62, 291A-304A.

12 P. Matousek, I. P. Clark, E. R. C. Draper, M. D. Morris, A. E. Goodship, N. Everall, M. Towrie, W. F. Finney and A. W. Parker, Appl. Spectrosc., 2005, 59, 393-400.

13 C. Conti, C. Colombo, M. Realini, G. Zerbi and P. Matousek, Appl. Spectrosc., 2014, 68, 686-691.

14 C. Conti, C. Colombo, M. Realini and P. Matousek, J. Raman Spectrosc., 2015, 46, 476-482.

15 P. Matousek, C. Conti, M. Realini and C. Colombo, Analyst, 2016, 141, 731-739.

16 B. B. Das, F. Liu and R. R. Alfano, Rep. Progr. Phys., 1997, 60, 227-292.

17 J. C. Hebden, A. Gibson, R. Yusof, N. Everdell, E. M. C. Hillman, D. T. Delpy, S. R. Arridge, T. Austin, J. H. Meek and J. S. Wyatt, Phys. Med. Biol., 2002, 47, 41554166.

18 H. Koizumi, Y. Yamashita, A. Maki, T. Yamamoto, Y. Ito, H. Itagaki and R. Kennan, J. Biomed. Opt., 1999, 4, 403413.

19 T. J. Pfefer, K. T. Schomacker, M. N. Ediger and N. S. Nishioka, Appl. Opt., 2002, 41, 4712-4721.

20 N. Ghosh, S. K. Majumder, H. S. Patel and P. K. Gupta, Opt. Lett., 2005, 30, 162-164.

21 L. Quan and N. Ramanujam, Opt. Lett., 2002, 27, 104-106.

22 S. C. Gebhart, S. K. Majumder and A. Mahadevan-Jansen, Appl. Opt., 2007, 46, 1343-1360.

23 C. Conti, M. Realini, C. Colombo and P. Matousek, Analyst, 2015, 140, 8127-8133.

24 K. Buckley, C. G. Atkins, D. Chen, H. G. Schulze, D. V. Devine, M. W. Blades and R. F. B. Turner, Analyst, 2016, 141, 1678-1685.

25 P. Matousek, M. D. Morris, N. Everall, I. P. Clark, M. Towrie, E. Draper, A. Goodship and A. W. Parker, Appl. Spectrosc., 2005, 59, 1485-1492.

26 M. Elias, Appl. Opt., 2011, 50, 2464-2473.

27 N. J. Elton and A. Legrix, J. Coat. Technol. Res., 2014, 11, 555-566.

28 P. Matousek and N. Stone, Chem. Soc. Rev., 2016, 45, 17941802. 